DOI: $10.22481 /$ recuesb.v8i14.7857

\title{
UTILIZAÇÃO DE VÍDEOS COMO APOIO SOCIAL PARA RESIDENTES DE INSTITUIÇÃO DE LONGA PERMANÊNCIA: RELATO DE EXPERIÊNCIA
}

\section{USE OF VIDEOS AS A SOCIAL SUPPORT FOR RESIDENTS OF A LONG STAY INSTITUTION: EXPERIENCE REPORT}

\author{
Erika Cristina Napolitano Giuliano ${ }^{1}$ \\ Bárbara Assis da Silva ${ }^{2}$ \\ Laura Campos dos Santos ${ }^{3}$ \\ Flávia Della Lucia ${ }^{4}$
}

\begin{abstract}
Resumo: Devido à pandemia da COVID-19 nas Instituições de Longa Permanência para Idosos, as visitas foram suspensas e a rotina alterada de modo a garantir a segurança de seus residentes. No entanto, tais medidas afetam negativamente os idosos devido a sensação de insegurança e a restrição do convívio com pessoas externas à instituição. Objetivo: Descrever a utilização de recursos de vídeo como forma de apoio social para os idosos residentes em uma Instituição de Longa Permanência para Idosos de um município do Sul de Minas Gerais durante o período de isolamento social causado pela pandemia da COVID-19. Metodologia: Trata-se de uma ação de extensão realizada pelo Projeto GerAções, componente da Universidade Aberta à Terceira Idade - UNATI em parceria com o Projeto NutrirArte. A ação consistiu na gravação de vídeos com mensagens de apoio e carinho a cada um dos idosos residentes da Instituição de Longa Permanência de uma cidade do Sul de Minas Gerais. Os vídeos foram gravados por membros dos dois projetos e enviados para a direção da Instituição que se encarregou de divulgá-los para os respectivos idosos. Resultados: Cada um dos 118 residentes recebeu um vídeo, sendo relatado pela direção da instituição que os idosos ficaram felizes e emocionados. Ainda de acordo com o relato da direção, assistir ao vídeo proporcionou apoio emocional, propiciando um alento à saúde psicológica dos residentes durante o período de isolamento social em decorrência da pandemia. Considerações finais: A realização dessa ação de extensão diminuiu o impacto negativo da suspensão das visitas presenciais na instituição durante o período de isolamento social. Ações semelhantes, que utilizem tecnologia para o
\end{abstract}

\footnotetext{
${ }^{1}$ Graduanda em Medicina, pela Universidade Federal de Alfenas (UNIFAL-MG), Alfenas, Minas Gerais, Brasil. Membro do projeto de extensão GerAções. E-mail: erikac.napolitano@gmail.com

${ }^{2}$ Graduanda em Medicina, pela Universidade Federal de Alfenas (UNIFAL-MG), Alfenas, Minas Gerais, Brasil. Membro do projeto de extensão GerAções. E-mail: baassis2000@ hotmail.com

${ }^{3}$ Graduanda em Fisioterapia, pela Universidade Federal de Alfenas (UNIFAL-MG), Alfenas, Minas Gerais, Brasil. Membro do projeto de extensão GerAções. E-mail: camposlaaura@gmail.com

${ }^{4}$ Nutricionista; Doutora em Ciência de Alimentos. Professora Adjunta da Faculdade de Nutrição, Universidade Federal de Alfenas, Alfenas, Minas Gerais, Brasil. Orcid: https://orcid.org/0000-0002-7585-2000 E-mail: flavia.lucia@unifal-mg.edu.br
} 
contato remoto e possibilitem o relacionamento intergeracional devem ser estimuladas.

Palavras-chave: Geriatria. Gerontologia. Universidade Aberta à Terceira Idade. Apoio Social.

Abstract: Due to the COVID-19 pandemic, in Long Term Care Institutions for the Elderly, visits were suspended and the routine was changed in order to ensure the safety of its residents. However, such measures affect negatively the elderly due to the feeling of insecurity and the restriction of being with people outside the institution. Objective: To describe the use of video resources as a form of social support for the elderly living in a Long Term Care Facility for the Elderly in a city in the south of Minas Gerais during the period of social isolation caused by the COVID-19 pandemic. Methodology: This is an extension action carried out by the GerAçoes Project, part of the Open University for the Third Age - UNATI in partnership with the NutrirArte Arte Project. The action consisted of recording videos with messages of support and affection for all elderly residents of the Long Term Care Institution in a city in the south of Minas Gerais. The videos were recorded by members of the two projects and sent to the management of the institution, which was in charge of disseminating them among the residents. Results: The receptivity and the return of the action were very satisfactory and offered social support in this very delicate moment, providing an encouragement to the residents' psychological health. Final considerations: It was possible to observe that actions like this are extremely important to promote better well-being and a better quality of life for the elderly living in a long-term institution and need to be disseminated and encouraged.

Keywords: Geriatrics. Gerontology. University Open to Seniors. Social Support.

\section{Introdução}

Em 31 de dezembro de 2019, foi notificado o primeiro caso de COVID-19 em Wuhan, na China, o vírus nomeado como Severe Acute Respiratory Syndrome Coronavirus-2 (SARSCoV-2) causa infecção aguda e, em 11 de março de 2020, foi declarada pandemia que alterou a rotina de todas as pessoas no mundo. Os dados apontam que a mortalidade pelo vírus é proporcionalmente maior com o aumento da idade, principalmente entre os que possuem doenças crônicas. A imunossenescência aumenta a vulnerabilidade dos idosos frente às doenças infectocontagiosas, o que somado às doenças crônicas, faz com que os prognósticos sejam piores nesses indivíduos. Entre os idosos com 80 anos ou mais a taxa de mortalidade é 14,8\% 
e nos idosos com idade entre 60 e 69 anos é 8,8\%. Já entre os residentes em ILPI, a taxa de mortalidade pela infecção pelo SARS-CoV-2 pode chegar a 15\%. Portanto, a preocupação com a população idosa frente esse novo contexto é justificável (HAMMERSCHMIDT; SANTANA, 2020).

Tendo em vista esse novo cenário, os serviços de assistência à pessoa idosa como as Instituições de Longa Permanência para Idosos (ILPI) precisaram sofrer mudanças e se readaptar. As ILPI são um ambiente de alto risco para disseminação da infecção, uma vez que os idosos residentes nessas instituições geralmente possuem múltiplas comorbidades, são dependentes dos funcionários para realização de muitas atividades básicas de vida diária, gerando um contato frequente e próximo com esses cuidadores, além do fluxo constante de profissionais, visitantes e da convivência em grupo, caracterizando aglomeração de pessoas (ROCHA; OLIVEIRA; MOTA, 2017).

Se houve mudança nas relações familiares entre os idosos não residentes em ILPI durante a pandemia de COVID-19, essa mudança foi ainda mais percebida entre aqueles que residiam nessas instituições. A Nota Técnica da Anvisa nº 05/2020 (ANVISA, 2020) trouxe novas orientações para a prevenção e o controle da COVID-19 em ILPI que alteraram profundamente a dinâmica dessas instituições e a relação social de seus residentes. A Nota Técnica reitera algumas intervenções incluindo: replanejar as rotinas de cuidados distribuindo atividades ao longo da jornada de trabalho para não haver aglomeração entre idosos e funcionários; suspender por tempo indeterminado as visitas e restringir as atividades em grupos; estabelecer um percurso de entrada para o funcionário da instituição de modo que somente tenha contato com os idosos após trocar a roupa com a qual chegou na ILPI por outra; evitar cumprimentos; realizar higiene das mãos dos profissionais e dos residentes; reduzir o tempo dos residentes nas áreas comuns da instituição para evitar aglomerações; realizar monitoramento periódico de todos os residentes quanto à febre, aos sintomas respiratórios e a outros sinais e sintomas da COVID-19; providenciar a utilização de máscaras cirúrgicas por idosos com sintomas de infecção respiratória sempre que estiverem fora dos quartos e possibilitar que idosos sintomáticos realizem atividades em horários diferentes dos outros

$$
\begin{array}{l|l}
\multirow{2}{*}{\text { REVISTA }} & \text { EXTENSÃO \& CIDADANIA } \\
\cline { 2 - 3 } & \text { v. 8, n. 14, p. 544-552, jul./dez. 2020. ISSN 2319-0566 }
\end{array}
$$


idosos, quando possível (ANVISA, 2020). Além disso, a Nota Técnica restringe a visita de profissionais que prestam serviços periódicos e voluntários como, por exemplo, cabeleireiros, podólogos e grupos religiosos (NUNES, 2020).

Assim, apesar da necessidade de se implementar ações preventivas específicas para idosos, direcionadas principalmente para o distanciamento social, ressalta-se o potencial deletério do isolamento imposto a esses indivíduos. Nesse sentido, é importante definir que o distanciamento social não é sinônimo de abandono do idoso, pois, no atual contexto, o afastamento físico é necessário para proteger a saúde de todos. Ou seja, para além de questões fisiopatológicas e epidemiológicas, deve-se discutir o impacto da pandemia de COVID-19 na saúde integral (HAMMERSCHMIDT; SANTANA, 2020), incluindo em relação ao apoio social percebido e à saúde mental dos idosos.

Quanto ao apoio social, entende-se que se trata de qualquer comportamento que tenha como objetivo assistir às necessidades de outra pessoa e que tenha efeitos positivos sobre a vida de quem recebe. $\mathrm{O}$ apoio social tem forte relação com o bem-estar subjetivo do indivíduo, principalmente do idoso, pois este sofre, na sua fase do ciclo vital, muitas perdas sociais (ROCHA; OLIVEIRA; MOTA, 2017). A assistência à saúde da pessoa idosa requer o apoio social, determinado pelos suportes instrumental, financeiro, emocional e relações sociais que as instituições ou pessoas podem oferecer. Dentre os benefícios do apoio social para a saúde do idoso estão: a melhoria dos parâmetros médicos, como a saúde mental, depressão e incapacidades; dos parâmetros sociais, como prevenção à institucionalização; dos parâmetros de saúde, como bem-estar e qualidade de vida. Assim, quando há uma participação social inadequada na vida do idoso, podem surgir quadros patológicos ou que contrariam as boas condições de vida; podendo até ser preditores de morte por distintas causas (GUEDES et al., 2017).

Durante a pandemia de COVID-19, várias estratégias foram usadas para tentar diminuir o isolamento causado pelo distanciamento social, como o uso de tecnologias. Porém, a população idosa tem muita dificuldade em usar tais recursos tecnológicos devido à frequente baixa escolaridade e à dificuldade de acesso. Tal fato dificulta tanto a aproximação virtual pela

$$
\begin{array}{l|l}
\multirow{2}{*}{\text { REVISTA }} & \text { EXTENSÃO \& CIDADANIA } \\
\cline { 2 - 3 } & \text { v. 8, n. 14, p. 544-552, jul./dez. 2020. ISSN 2319-0566 }
\end{array}
$$


comunicação com áudio ou vídeo-chamadas como para que obtenham informações acerca da pandemia (HAMMERSCHMIDT; SANTANA, 2020).

Dessa forma a ação desenvolvida pelo projeto de Extensão GerAções juntamente com o projeto NutrirArte teve como objetivo estimular o apoio social vivenciado pelos idosos residentes em uma instituição de longa permanência do Sul de Minas Gerais, na intenção que estes sentissem um pouco menos o impacto do distanciamento social e com a ajuda dos profissionais da instituição fosse reduzida a dificuldade de acesso desses idosos a contatos virtuais.

\section{Metodologia}

Trata-se de um relato de experiência sobre uma ação de extensão realizada no âmbito do Projeto de Extensão GerAções, componente do Programa de extensão da Universidade Aberta à Terceira Idade - UNATI, juntamente com o Projeto de Extensão NutrirArte, componente do Programa de Extensão Agroecologia, Educação Ambiental e Soberania Alimentar, ambos da Universidade Federal de Alfenas - UNIFAL-MG.

O projeto GerAções é desenvolvido pela Liga Acadêmica de Geriatria e Gerontologia da UNIFAL-MG e tem como objetivo desenvolver aulas abertas, oficinas de formação e atividades de promoção do envelhecimento ativo e saudável junto à população e/ou comunidade acadêmica, visando à disseminação e ao aprofundamento dos conhecimentos atuais na área da geriatria e gerontologia. Já o projeto NutrirArte realiza ações de educação nutricional e promoção de saúde de forma lúdica para a comunidade.

A ação se desenvolveu no período de abril a maio de 2020 durante a pandemia de COVID-19, no município de Alfenas, Minas Gerais, por meio da confecção de vídeos individuais e personalizados para cada um dos 118 idosos residentes em uma Instituição de Longa Permanência. Tais vídeos foram gravados por 15 discentes e 3 docentes membros de ambos os projetos, em suas residências, utilizando aparelhos celulares ou notebooks. Foi padronizado para o conteúdo do vídeo a apresentação do narrador e a utilização do nome do

$$
\begin{array}{l|l}
\multirow{2}{*}{\text { REVISTA }} & \text { EXTENSÃO \& CIDADANIA } \\
\cline { 2 - 3 } & \text { v. 8, n. 14, p. 544-552, jul./dez. 2020. ISSN 2319-0566 }
\end{array}
$$


idoso residente que iria receber aquele determinado vídeo, como forma de personalizar a mensagem. O restante do conteúdo foi deixado com tema livre para que os membros criassem as mensagens (poemas, músicas, orações). O conteúdo criado não levou em consideração características específicas dos idosos, tais como, religiosidade, preferência musical, etc. Os vídeos tinham entre 2 e 3 minutos e foram enviados para a direção da Instituição que, posteriormente, reproduziu os vídeos para cada idoso.

\section{Resultados e discussão}

No momento da realização da ação, 118 idosos residiam na instituição, sendo que cada um recebeu um vídeo. Os resultados da ação foram analisados qualitativamente utilizando os relatos dos funcionários da instituição, sendo recorrente nos discursos a percepção de que tanto os idosos quanto os funcionários que reproduziram os vídeos ficaram felizes e emocionados. Apreendeu-se das falas dos funcionários que a ação teve receptividade e retorno satisfatórios, o que permite inferir que os vídeos foram uma importante ferramenta de provisão de apoio emocional neste momento tão delicado, propiciando um alento à saúde psicológica dos idosos residentes.

O distanciamento social não deve significar o abandono destes residentes que continuam necessitando de apoio e a iniciativa permitiu que os idosos se sentissem acolhidos, importantes e menos tristes frente à situação atual (distância dos familiares, amigos, entre outras pessoas significativas).

A pandemia de COVID-19 mudou muitos aspectos no mundo, incluindo como os idosos são tratados. O discurso público, veiculado pelas mídias sociais, imprensa e pronunciamentos políticos, descreve aqueles com mais de 70 anos como iguais em relação à impotência, à fragilidade e à incapacidade de contribuir com a sociedade, mas, o processo de envelhecimento é muito diversificado e dependente do contexto individual. Inúmeros idosos desafiam a imagem de fragilidade e desamparo, fazendo valiosas contribuições para a sociedade. Sabemos que estereótipos negativos de idade podem ser internalizados por pessoas de todas as idades e

$$
\begin{array}{l|l}
\multirow{2}{*}{\text { REVISTA }} & \text { EXTENSÃO \& CIDADANIA } \\
\cline { 2 - 3 } & \text { v. 8, n. 14, p. 544-552, jul./dez. 2020. ISSN 2319-0566 }
\end{array}
$$


quando essas visões se tornam relevantes, influenciando crenças dos idosos sobre seu próprio envelhecimento, elas podem afetar negativamente a saúde (AYALON et al., 2020).

Na tentativa de proteger a saúde dos idosos por meio do distanciamento social, não se deve abandoná-los, nem ter preconceito com eles, trazendo à tona o importante conceito de ageísmo. O Ageísmo pode ser definido como estereótipo, preconceito ou discriminação contra as pessoas com base na idade. Refere-se a quão negativo crenças, atitudes, suposições e estereótipos sobre a idade são usados para discriminazar e marginalizar os idosos (HARRIS et al., 2017).

Portanto, o distanciamento físico não precisa provocar um distanciamento emocional. Assim que o contato intergeracional se torna individualizado (por exemplo, nas famílias, contatos pessoais com vizinhos mais velhos), em vez de tomar lugar no discurso público, os efeitos negativos da categorização social, estigmatização, preconceito e estereótipos são fortemente reduzidos e substituídos por normas de solidariedade e parentesco (AYALON et al., 2020). Promover essa forma de contato pessoal entre idosos e jovens é um meio importante de superar as tensões intergeracionais e foi o objetivo desta iniciativa. Os discentes, de vários cursos da área de saúde, ao participarem de atividades como essa puderam experienciar a importância do apoio social. Desta forma, a sua participação pode consolidar ainda mais sua formação profissional, conseguindo desta forma perceber a importância do profissional de saúde em ações não somente de prevenção e promoção, mas também de proteção e reabilitação da saúde, tanto em nível individual quanto coletivo.

Além dos benefícios emocionais, já foi verificada, em estudos epidemiológicos, a relação entre um apoio social ao idoso adequado e efetivo e a menor incidência de hipertensão arterial sistêmica (HAS) e risco de demência, maior chance de sobrevivência após diagnóstico de acidente vascular cerebral (AVC), neoplasias, doença coronariana e uma comprovada menor mortalidade geral (AMARAL et al., 2013).

Há diversas dimensões dentro do apoio social com relação ao seu conteúdo: o instrumental é aquele em que se fornece recursos materiais ou assistência prática em atividades concretas; o emocional envolve comportamentos que valorizam o outro e o informacional é

$$
\begin{array}{l|l}
\multirow{2}{*}{\text { REVISTA }} & \text { EXTENSÃO \& CIDADANIA } \\
\cline { 2 - 3 } & \text { v. 8, n. 14, p. 544-552, jul./dez. 2020. ISSN 2319-0566 }
\end{array}
$$


aquele em que se fornece informações ou conselhos úteis (ROCHA; OLIVEIRA; MOTA, 2017). Os vídeos desenvolvidos pelos discentes dos projetos GerAções e NutrirArte, tiveram como objetivo o provimento de apoio emocional para os residentes da ILPI, para que esses se sentissem queridos, valorizados e pertencentes à comunidade.

\section{Considerações finais}

A experiência de realização dessa ação de extensão universitária reforçou a importância de se desenvolver ações destinadas à idosos residentes em ILPI, uma vez que não foram encontradas publicações sobre experiências semelhantes e que tais instituições enfrentam muitos desafios em relação ao isolamento social dos residentes, que se acentuaram com a pandemia de COVID-19. Iniciativas que promovam bem-estar e uma melhor qualidade de vida às pessoas idosas e precisam ser divulgadas e estimuladas tendo em vista o bem estar social de quem recebe e de quem promove as ações.

A facilidade de acesso à internet e de utilização de ferramentais digitais para produzir os vídeos por parte dos discentes e docentes contribuiu para o sucesso da ação. Além disso, essa ação demonstrou excelente relação custo-benefício, o que justifica que se estimule que ações semelhantes sejam desenvolvidas continuamente nas instituições, a fim de proporcionar apoio social e troca intergeracional aos idosos que vivem em regime de internato. No mais, o contato remoto pode estimular o desenvolvimento de ações de promoção da inclusão digital dos idosos. 


\section{Referências}

ANVISA. Agência Nacional de Vigilância Sanitária. Nota Técnica VIMS/GGTES/ANVISA n⿳0 05/2020: Orientações para a prevenção e o controle de infecções pelo novo coronavírus (SARS-CoV-2) em instituições de longa permanência para idosos (ILPI) Brasília: Anvisa; 2020.

AMARAL, Fabienne Louise Juvêncio dos Santos et al. Perfil do apoio social de idosos no município de Natal, Estado do Rio Grande do Norte, Brasil, 2010-2011. Revista Epidemiologia e Serviços de Saúde, Brasília , v. 22, n. 2, p. 335-346, jun. 2013.

AYALON, Liat et al. Aging in Times of the COVID-19 Pandemic: avoiding ageism and fostering intergenerational solidarity. Journals of Gerontology: Psychological Sciences, v. XX, n. XX, p. 1-4 April 16, 2020. Disponível em:

https://academic.oup.com/psychsocgerontology/advance-

article/doi/10.1093/geronb/gbaa051/5820621. Acesso em: 17 out. 2020.

GUEDES, Marcelo Barbosa Otoni Gonçalves et al. Apoio social e o cuidado integral à saúde do idoso. Physis, Rio de Janeiro, v. 27, n. 4, p. 1185-1204, dez. 2017.

HAMMERSCHMIDT, Karina Silveira de Almeida; SANTANA, Rosimere Ferreira. Saúde do idoso em tempos de pandemia Covid-19. Cogitare Enfermagem, [S.L.], v. 25, p. 1-10, 2020.

HARRIS, Kelly et al. Ageism and the Older Worker: a scoping review. The

Gerontologist, [S.L.], Oxford University Press (OUP), p. 1-14, jan. 2017. Disponível em: https://academic.oup.com/gerontologist/article/58/2/e1/2894393. Acesso em: 18 out. 2020.

NUNES, Vilani Medeiros de Araújo et al. COVID-19 e o cuidado de idosos: recomendações para instituições de longa permanência. Natal: EDUFRN, 2020.

ROCHA, Luiz Fellipe Dias da; OLIVEIRA, Evlyn Rodrigues; MOTA, Marcia Maria Peruzzi Elia da. Relação entre apoio social e bem-estar subjetivo em idosos: revisão sistemática. Revista Brasileira em Promoção da Saúde, [S.L.], v. 30, n. 4, p. 1-13, 6 dez. 2017.

Recebido em: 20 de outubro de 2020.

Aceito em: 5 de dezembro 2020. 